\title{
Perspectiva de estudantes de Farmácia sobre aprendizagem experiencial na Atenção Primária à Saúde: o PET-Saúde/GraduaSUS
}

\author{
Pharmacy students` perspective of experiential learning in Primary Health Care: the PET-
}

\author{
Health/GraduaSUS
}

Perspectiva de los estudiantes de Farmacia sobre aprendizaje experiencial na Atención Primaria de Salud: el PET-Salud/GraduaSUS

\section{Resumo}

Objetivo: O objetivo com o presente estudo foi compreender a perspectiva dos estudantes de Farmácia sobre aprendizagem experiencial na Atenção Primária à Saúde por meio do projeto PET-Saúde/GraduaSUS, no período de dois anos (2016/2018). Métodos: Tratou-se de um estudo de caso, empregando-se métodos de pesquisa qualitativa. Os participantes do estudo foram estudantes de Farmácia que integraram o projeto PET-Saúde/GraduaSUS entre os anos de 2016 a 2018. Foram realizadas entrevistas semiestruturadas abordando o impacto do projeto na vida dos estudantes, aspectos positivos e dificuldades, e sugestões para a melhoria da atividade. Os dados foram submetidos à análise de conteúdo. Resultados: Emergiram cinco categorias dos relatos dos graduandos: Conhecimento sobre o sistema de saúde, Atuação em equipe interprofissional de saúde, Formação de identidade profissional farmacêutica, Proposição e realização de ações de acordo com as necessidades da população, Habilidade de realizar ações de educação em saúde. Conclusão: Os relatos sugeriram que os objetivos de aprendizagem propostos para os estudantes de Farmácia foram alcançados. O Projeto PET-Saúde possibilitou a oportunidade de formação no âmbito interprofissional e na atenção à saúde.

Palavras-chave: Atenção primária à saúde; Assistência farmacêutica; Educação em saúde; Educação interprofissional; Educação em farmácia.

\footnotetext{
Abstract

Objective: The aim with the current study was to understand Pharmacy undergraduates' perspective on experiential learning in Primary Health Care through PET-Health/GraduaSUS project, in a period of two years (2016/2018). Methods: It was a case study, carried out through methods of qualitative research. The study participants were Pharmacy students that took part in the project PET-Health/GraduaSUS from 2016 to 2018. Semi structured interviews were carried out addressing the impact of the project on students` life, positive and negative aspects and suggestions to improve the activity. A content analysis of the data was performed. Results: Five categories emerged from students` reports: Knowledge about the health system, Performance in interprofessional health team, Formation of pharmacist`s professional identity, Proposal and performance of actions addressed to population health needs, Skill to perform actions of health education. Conclusion: The reports suggested that the learning objectives proposed to the
} 
Pharmacy students were achieved. The PET-Health/GraduaSUS project enabled the opportunity of formation in an interprofessional context and in health care.

Keywords: Primary health care, Pharmaceutical services, Health education, Interprofessional education, Pharmaceutical education.

\section{Resumen}

Objetivo: El objetivo con este estudio fue comprender la perspectiva de los estudiantes de Farmacia sobre aprendizaje experiencial en Atención Primaria de Salud, por medio del proyecto PET-Salud/GraduaSUS, durante un período de dos años (2016/2018). Métodos: Fue un estudio de caso, utilizando métodos de investigación cualitativos. Los participantes del estudio fueron estudiantes de Farmacia que formaron parte del proyecto PET-Salud/GraduaSUS entre los años 2016 a 2018. Se realizaron entrevistas semiestructuradas abordando el impacto del proyecto en la vida de los estudiantes, aspectos positivos y dificultades y sugerencias para la mejora de la actividad. Un análisis de contenido de los datos fue realizado. Resultados: De los informes de los estudiantes surgieron cinco categorías: Conocimiento sobre el sistema de salud, Trabajo en equipo interprofesional de salud, Formación de la identidad farmacéutica profesional, Proponer y realizar acciones de acuerdo a las necesidades de la población, Capacidad para realizar acciones de educación en salud. Conclusión: Los informes sugirieron que se alcanzaron los objetivos de aprendizaje propuestos para los estudiantes de Farmacia. El Proyecto PET-Salud brindó la oportunidad de formación interprofesional y asistencial.

Palabras clave: Atención primaria de salud; Servicios farmacéuticos; Educación en salud; Educación interprofesional; Educación en farmacia.

\section{Introdução}

Em todo o mundo observam-se que as condições de saúde estão se tornando cada vez mais complexas diante de mudanças sociodemográficas, epidemiológicas e até mesmo culturais (Filho et al., 2019). A complexidade das ações desenvolvidas em um ambiente clínico exige ações conjuntas com profissionais da saúde, de forma a desenvolver a prática colaborativa. A prática interprofissional colaborativa diz respeito à colaboração expressa no cenário de prática, ou seja, a interprofissionalidade no âmbito do trabalho em saúde. Existe uma valorização do cuidado integral em saúde e da atenção centrada no paciente, incluindo a família, cuidadores e comunidade no processo de tomada de decisão, princípios básicos da Atenção Primária à Saúde (APS) no Sistema Único de Saúde (SUS) (Brasil, 2017). Desta forma, levar o paciente para o centro do cuidado faz com que seja necessário abrir os horizontes para além das especificidades de cada profissão, estimulando a interação entre as diferentes profissões com o objetivo de obter uma atenção à saúde de melhor qualidade (Agreli et al., 2019).

Os farmacêuticos, enquanto profissionais da saúde responsáveis pelas necessidades relacionadas aos medicamentos, devem possuir atitudes colaborativas de forma a dialogar com a equipe de saúde para se ter uma visão geral do paciente, com o objetivo de proporcionar maior integralidade do cuidado, garantindo assim, a melhoria da qualidade de vida do paciente (FIP, 2012). Assim, a educação interprofissional (EIP) é essencial para o desenvolvimento de habilidades colaborativas na formação dos farmacêuticos. A EIP é uma oportunidade de aprender e interagir com estudantes e membros do corpo docente de outros programas profissionais de saúde e melhorar a qualidade dos serviços e seus resultados, principalmente em termos de segurança dos pacientes (Reeves, 2016). A aprendizagem experiencial tem sido descrita como um método efetivo para alcance dos objetivos propostos pela EIP. Ela se baseia na busca do conhecimento do processo da aprendizagem baseada na própria experiência, em que o aprendizado não se dá somente por processos cognitivos, mas também pelo afeto, pela percepção e pela ação (Kolb, 2015). Um programa educacional que almeje levar o estudante a alcançar uma consciência integrativa deve expor o mesmo à sua prática profissional, amparado por instrumentos que permitam a reflexão e promovam a compreensão do que está sendo vivenciado.

No Brasil, as discussões acerca do trabalho em equipe e da formação dos profissionais de saúde estão em pauta desde que surgiram os primeiros movimentos em prol da construção do sistema nacional de saúde, por volta das décadas de 1960 e 1970. Com o apoio de instâncias governamentais e da cooperação internacional, o país apresentou uma série de iniciativas com 
o objetivo de modificar o modelo tradicional de formação uniprofissional e, consequentemente, alterar o modelo fragmentado de atenção à saúde (Filho et al., 2019; Silva et al., 2015; Peduzzi et al., 2013).

Dentre algumas ações propostas, em 2005 foi criado o Programa Nacional de Reorientação da Formação Profissional em Saúde (Pró-Saúde), que trouxe como temática principal a integração entre ensino e serviço através da inserção dos estudantes nos cenários de prática (Peduzzi et al., 2013). Uma das estratégias para seu fortalecimento foi o Programa de Educação pelo Trabalho em Saúde (PET-Saúde), que teve como objetivo a criação de grupos tutoriais compostos por profissionais inseridos no serviço, estudantes e docentes para promover a aprendizagem. Os grupos tutoriais são inseridos em locais estratégicos, sendo que cada edital lançado possui uma temática específica e é uma importante estratégia para transformar a formação dos profissionais de saúde e qualificar a Atenção Primária à Saúde (Leite et al., 2012). Vários estudos mostraram que o PET-Saúde proporcionou aos estudantes experiência na APS e no trabalho interprofissional em saúde (Mangueira et al., 2021; Rosa et al., 2015; Lima et al., 2014, Mendes et al., 2014; Barreto et al., 2011). Por meio do programa, os estudantes têm a oportunidade de perceberem sua importância e papel na sociedade como profissionais da saúde, perceber os limites profissionais e aprender com as equipes de trabalho. Em 2016 foi lançado o edital para o PET-Saúde/GraduaSUS, que visava a promoção de mudança na graduação dos cursos da área da saúde, com ampliação do SUS enquanto espaço de aprendizagem, corresponsabilização das Instituições de Ensino Superior pela saúde no território, desenvolvimento dos trabalhadores do SUS como protagonistas da integração ensino-serviço-comunidade e o fomento da educação permanente em saúde (Brasil, 2015).

Assim, o presente trabalho se propôs a compreender a perspectiva dos estudantes de Farmácia sobre a aprendizagem experiencial na Atenção Primária à Saúde por meio do projeto PET-Saúde/GraduaSUS.

\section{Metodologia}

\section{Delineamento metodológico}

Tratou-se de um estudo de caso, empregando-se métodos de pesquisa qualitativa. O estudo de caso é um tipo de pesquisa que visa examinar em detalhes fenômenos da vida real, como experiências de vida, fenômenos sociais, instituições e indivíduos (Turhan, 2019). Para o presente estudo, optou-se pelo estudo de caso para conhecer com detalhamento as experiências dos estudantes com o PET-Saúde/GraduaSUS e o significado atribuído a elas pelos próprios alunos.

\section{Contexto da atividade educativa}

No município de Belo Horizonte, Minas Gerais, o programa PET-Saúde/GraduaSUS deu-se por meio de uma parceria entre a Universidade Federal de Minas Gerais (UFMG) e a Secretaria Municipal de Saúde de Belo Horizonte (SMSA-BH), no período de maio de 2016 a abril de 2018. Seis grupos tutoriais com estudantes de cursos de graduação da saúde da Universidade Federal de Minas Gerais atuaram nos serviços da SMSA-BH, e entre eles estava incluído o curso de Farmácia, que nesta universidade conta com dez períodos no turno diurno e treze períodos no turno noturno. As ações de integração ensino-serviço-comunidade do grupo do Pet-Saúde/GraduaSUS com estudantes de Farmácia apresentaram como foco a promoção da saúde e do uso racional de medicamentos. A maioria das ações foi realizada em conjunto com estudantes e profissionais de saúde de outras áreas diferentes da Farmácia, como Enfermagem, Medicina, Nutrição, Fisioterapia e Educação Física. O grupo tutorial de Farmácia foi composto por cinco graduandos, três docentes tutoras e cinco preceptoras da SMSA$\mathrm{BH}$, sendo destas quatro farmacêuticas e uma enfermeira. As preceptoras farmacêuticas eram vinculadas a equipes do Núcleo de Apoio à Saúde da Família (NASF). Os cenários de prática do grupo no SUS foram oito Unidades Básicas de Saúde (UBS) da SMSA-BH. 
Os objetivos de aprendizagem propostos pelos docentes tutores para os graduandos de Farmácia incluíram diagnosticar a situação de saúde da população adscrita aos serviços de saúde, propor e executar ações voltadas para as necessidades dos serviços de saúde e atuar de forma interprofissional focada na humanização do cuidado e na integralidade da assistência. O desenvolvimento dos métodos de aprendizagem pelos docentes tutores do grupo de Farmácia para o projeto foi baseado na aprendizagem experiencial de Kolb (Kolb, 2015) e na formação por competências, em que é desenvolvida a capacidade do educando de executar ações em um cenário da vida real (Frank et al., 2010). Nos primeiros três meses do programa, os graduandos realizaram a observação das atividades realizadas nas UBS, registrando e compartilhando com o grupo tutorial suas reflexões, análise crítica e sugestões para o aprimoramento da APS. Durante a execução do projeto, os alunos e preceptores preenchiam quinzenalmente os diários de campo, com o registro das atividades planejadas, executadas e as reflexões sobre as mesmas. Mensalmente, eram realizadas reuniões com todos os membros do grupo (graduandos, preceptores e tutores) para compartilhar as percepções sobre as atividades realizadas e planejamento conjunto das ações a serem executadas.

\section{Participantes do estudo}

Os participantes do estudo foram graduandos de Farmácia da Universidade Federal de Minas Gerais (UFMG), participantes do PET-Saúde/GraduaSUS em Belo Horizonte que atuaram entre maio de 2016 a março de 2018 , durante um período de no mínimo seis meses. Foram incluídos oito estudantes, sendo três do sexo masculino e cinco do sexo feminino. Todos os alunos desenvolveram suas atividades em UBS, sendo que seis tinham como preceptor um farmacêutico e dois, um enfermeiro. Ao ingressar no projeto, cinco estudantes cursavam até o quinto período do curso e os demais encontravam-se entre o sétimo e o nono período.

\section{Coleta de dados}

Para as entrevistas dos estudantes, foi desenvolvido um roteiro estruturado constituído por perguntas sobre o impacto do programa na vida dos estudantes, aspectos positivos e dificuldades e sugestões para a melhoria da atividade. Os itens foram:

1) O que mudou na sua vida com a participação no PET-Saúde/GraduaSUS?

2) Comente os pontos positivos da experiência no PET-Saúde/GraduaSUS.

3) Comente as dificuldades encontradas na experiência no PET-Saúde/GraduaSUS.

4) Quais sugestões você daria para a melhoria do Programa PET-Saúde/GraduaSUS?

As entrevistas foram realizadas e gravadas pela coordenadora do grupo tutorial de Farmácia de maio a junho de 2018. A graduanda em Farmácia principal autora do presente trabalho, que não participou do PET-Saúde/GraduaSUS, transcreveu integralmente os áudios das entrevistas realizadas com os participantes. Os dados das entrevistas foram digitados em um banco de dados no Microsoft Excel. Foi atribuído um número a cada um dos oito alunos para preservar a confidencialidade dos seus dados.

\section{Análise dos dados}

As transcrições dos relatos dos participantes foram analisadas diversas vezes por meio da técnica de leitura flutuante com o objetivo de compreender de forma ampla os significados expressos nas falas dos participantes. A análise categorial foi 
escolhida por ser uma alternativa apropriada para estudar valores, opiniões e atitudes através de dados qualitativos. As categorias foram identificadas conforme proposto por Bardin (2002), por meio de seleção do material, leitura flutuante e classificadas de acordo com a similaridade dos assuntos. A classificação dos relatos em categorias foi realizada pela principal autora do presente trabalho. Posteriormente, outra autora revisou as categorias e foi obtido consenso em caso de divergências.

\section{Aspectos éticos}

O projeto de pesquisa foi aprovado pelo Comitê de Ética em Pesquisa da Universidade Federal de Minas Gerais e da Secretaria Municipal de Saúde de Belo Horizonte (CAAE 79472017.8.0000.5149). Os oito estudantes que integraram o PETSaúde/GraduaSUS durante um período de no mínimo seis meses foram convidados para participar da presente pesquisa e todos concordaram. Todos os entrevistados que consentiram em participar da pesquisa assinaram o Termo de Consentimento Livre e Esclarecido (TCLE).

\section{Resultados e Discussão}

Após a análise de dados, emergiram cinco categorias dos relatos dos graduandos: Conhecimento sobre o sistema de saúde, Atuação em equipe interprofissional de saúde, Formação de identidade profissional farmacêutica, Proposição e realização de ações de acordo com as necessidades da população e Habilidade de realizar ações de educação em saúde. Os relatos sugeriram que os objetivos de aprendizagem propostos para os estudantes do grupo tutorial de Farmácia foram alcançados.

\section{Categoria: Conhecimento sobre o sistema de saúde}

Relatos dos estudantes mostraram que muitos alteraram a percepção a respeito do SUS após a participação no projeto. Por meio da mídia ou troca de informações com pessoas do convívio social, muitas vezes, tinham um olhar apenas negativo do SUS e com a participação no projeto, conseguiram desmistificar e identificar ações positivas que são efetuadas diariamente pelos profissionais. A experiência com o Programa PET-Saúde/GraduaSUS permitiu aos participantes que construíssem uma visão crítica acerca do sistema, além do aprendido nas salas de aula. Os alunos puderam conhecer a visão dos usuários sobre o SUS e comparar a forma como o sistema é retratado nas mídias com a forma que eles mesmos vivenciaram nas UBS.

A experiência e contato com farmacêuticos e profissionais de outras áreas da saúde foi, na percepção dos alunos, importante para agregar conhecimento além do teórico adquirido durante a graduação. A participação no projeto proporcionou também que os estudantes visualizassem a integração entre o paciente e os profissionais, como essa relação é importante e como o foco é centrado quase em sua totalidade no bem-estar do paciente.

[...] E uma vez presente no centro de saúde eu consegui ter essa visualização, essa integração entre o paciente e o profissional, o quanto o profissional é importante para esse paciente e que esse paciente representa (Aluno 2 em trecho de entrevista)

Segundo um estudo realizado sobre uma iniciativa dentro do PET-Saúde na Universidade de São Paulo, foi mencionado que este programa foi um dispositivo capaz de proporcionar uma melhor percepção acerca do SUS, especialmente da APS, quebrando ideias e opiniões desfavoráveis (Fonseca et al., 2014). 


\section{Categoria: Atuação em equipe interprofissional de saúde}

A oportunidade de atuar com estudantes e profissionais de saúde de diferentes categorias proporcionou aos graduandos de Farmácia o desenvolvimento de habilidades e atitudes de atuação em equipe interprofissional de saúde. Eles ressaltaram a potencialidade do trabalho em equipe para atender às necessidades de saúde da população e para a sua própria formação profissional. Ocorreu, de acordo com os relatos dos discentes, uma troca de conhecimentos única, pois é uma experiência que não acontece de forma tão pronunciada durante a graduação.

No início do projeto os alunos relataram desconfiança dos profissionais da equipe sobre a atuação deles. Entretanto, com o decorrer das atividades, a atuação dos discentes, segundo percepções dos mesmos, foi considerada como positiva pelos profissionais da UBS, que passaram a estar mais dispostos para a prática colaborativa interprofissional.

[...] Depois com a convivência dentro do centro de saúde acho que eles [os profissionais] acostumaram com nossa presença e começaram a nos ver mais assim como colegas e não como uma pessoa que está ali para apontar o dedo literalmente (Aluno 2 em trecho de entrevista).

Segundo um estudo realizado em um município do estado de Pernambuco, a experiência com o Pet-Saúde foi um processo de construção coletiva no qual os envolvidos precisaram necessariamente desenvolver habilidades colaborativas essenciais para o trabalho em saúde, tais como: escuta, comunicação, iniciativa, proatividade, sensibilidade e aprimoramento técnico (Mangueira et al., 2021).

Ao longo do projeto PET-Saúde/GraduaSUS, os estudantes de Farmácia passaram a se sentir como membros da equipe de saúde, vislumbrando a contribuição de diferentes profissionais no processo de saúde-doença da população e tendo a sua própria contribuição reconhecida pelos trabalhadores, estudantes e usuários dos serviços.

[...] Quando a gente propõe alguma coisa eles [os profissionais] são os primeiros que falam que querem fazer, principalmente grupos operativos, assim eu tive muita sorte, mas todos queriam colocar em prática, se propuseram a ajudar, a disponibilizar horário, e tudo mais (Aluno 2 em trecho de entrevista)

[...] Outros profissionais como nutricionista, terapia [ocupacional] vieram me perguntar algumas coisas da farmácia quando a [farmacêutica] não estava. Então assim foi bem, foi uma experiência muito boa, agregou muito conhecimento tanto profissional quanto pessoal (Aluno 8 em trecho de entrevista)

Estudos internacionais que avaliaram intervenções clínicas realizadas por estudantes de Farmácia durante iniciativas de aprendizagem experiencial ilustraram que as ações dos graduandos foram bem aceitas pela equipe interprofissional de saúde (Kwon et al., 2021; Mead et al., 2017).

\section{Categoria: Formação de identidade profissional farmacêutica}

O acompanhamento do trabalho do farmacêutico na APS e o desenvolvimento de atividades profissionais nos serviços de saúde proporcionaram aos estudantes de Farmácia o início da formação de uma identidade profissional farmacêutica. Os graduandos tiveram com o PET-Saúde/GraduaSUS a possibilidade de aplicar o conhecimento sobre medicamentos e de desenvolver habilidades clínicas e de comunicação com os usuários. Eles relataram, também, que não tiveram esta oportunidade ao longo do curso de Farmácia. A maioria dos alunos se encontrava na primeira metade do curso de Farmácia. Nas entrevistas, eles relataram os benefícios desta inserção precoce na APS e o desenvolvimento de competências para a 
prática profissional farmacêutica. Um estudante relatou que teve melhores oportunidades profissionais após participar no programa.

[...]Por causa do PET, eu tenho certeza que abriu portas para mim em outros estágios, em algumas entrevistas que eu fiz, você sabe que você não está cru (Aluno 4 em trecho de entrevista)

Nas últimas décadas em vários países do Mundo, a profissão farmacêutica evoluiu de uma lógica centrada no medicamento para uma lógica centrada no paciente. Os cursos de Farmácia acompanharam esta mudança, procurando o desenvolvimento de habilidades nos estudantes para acompanhar este novo papel do farmacêutico. Em países como Estados Unidos, Austrália e Nova Zelândia, por exemplo, a formação do farmacêutico tem como foco o desenvolvimento de competências de atenção ao paciente por meio da aprendizagem experiencial (Kairuz et al., 2010; Marriott et al., 2008; Nemire \& Meyer, 2006). No Brasil, o ensino farmacêutico é ainda baseado nas ciências básicas e farmacêuticas, com poucas vivências de aprendizagem experiencial no cuidado ao paciente (Mendonça et al., 2017). Esta situação pode ter explicado o fato dos alunos do PET-Saúde/GraduaSUS não terem tido a oportunidade de contato com o paciente antes de ingressar no programa.

A participação no PET-Saúde/GraduaSUS proporcionou aos estudantes a oportunidade de conhecer a rotina do farmacêutico na APS e suas dificuldades. Segundo os alunos entrevistados, o fato dos preceptores farmacêuticos atuarem em duas UBS diferentes com atividades de natureza distintas prejudicou a fluidez das ações do PET-Saúde. Estas dificuldades apontadas pelos estudantes têm relação com a forma de inserção dos farmacêuticos na APS em Belo Horizonte no momento do programa, vinculados a uma equipe do Núcleo de Apoio à Saúde da Família (NASF). No NASF em Belo Horizonte, o farmacêutico divide sua carga horária de trabalho como provedor de serviços clínicos farmacêuticos e das atividades técnico gerenciais (Destro et al., 2021).

Apesar das dificuldades na preceptoria, um estudante relatou a importância do preceptor para a sua formação ao longo do programa.

[...] ] farmacêutica ela me auxiliou muito bem em tudo que eu precisava, me respondeu e tudo mais, tirou minhas dúvidas, passou muita experiência dela também (Aluno 4 em trecho de entrevista).

\section{Categoria: Proposição e realização de ações de acordo com as necessidades da população}

A inserção em uma realidade diferente da qual os estudantes estão acostumados foi capaz de auxiliar na identificação das dificuldades dos serviços de saúde e contribuir para a procura de melhorias e alternativas. Os alunos identificaram que são necessárias diversas mudanças, mas que eles podem agregar auxiliando e atuando como agentes transformadores no sistema de saúde. No decorrer do projeto, os estudantes se sentiram seguros para propor ações de acordo com as necessidades da população e dos serviços de saúde.

[...]A questão do amadurecimento também que ao longo do PET, eu cheguei no meio do PET a ficar mais atrás de uma colega [graduanda de Farmácia do PET-Saúde], assim e agora eu tomo mais iniciativa para fazer algumas atividades (Aluno 7 em trecho de entrevista).

Por terem sido expostos às atividades das UBS, refletirem sobre as mesmas e relacioná-las com a literatura técnicocientífica, os estudantes de Farmácia do presente do estudo puderam propor ações. Desta forma, eles atingiram todos os níveis da aprendizagem experiencial, incluindo a experiência concreta; a observação reflexiva; a conceitualização abstrata, em que a 
pessoa é capaz de criar conceitos que integrem suas observações em teorias lógicas e a experimentação ativa, em que a pessoa é capaz de usar essas teorias para tomar decisões e resolver problemas (Kolb, 2015; Pimentel, 2007). Segundo estudos realizados com estudantes em iniciativas do PET-Saúde, eles propuserem ações para a promoção da saúde da população adscrita às UBS após exposição e reflexão da prática na APS (Lima et al., 2014; Mendes et al., 2014).

Por meio do PET-Saúde, os acadêmicos tiveram a oportunidade de aplicar os conhecimentos teóricos nas experiências práticas, além do contato direto com a comunidade e outros profissionais de saúde para situar os alunos da importância de seu papel como futuro profissional da saúde e a diferença que podem fazer na vida dos pacientes, conhecendo a realidade da vida deles e contribuir para uma intervenção positiva. Os graduandos desenvolveram ações que contribuíram para a promoção da saúde da população e do uso racional de medicamentos, como atividades educativas e de acompanhamento farmacoterapêutico. A satisfação com esta contribuição foi relatada por um estudante:

[...] Porque passa para a gente que não tem nada, que não dá para fazer nada, mas se a gente quiser dá para fazer. Mesmo se for pouca coisa, qualquer coisa que você faça para mudar um pouquinho a percepção de um medicamento ou de uma doença, de um paciente que precisa, já é alguma coisa, então isso mudou muito para mim (Aluno 5 em trecho de entrevista).

Os estudantes relataram dificuldades em propor e realizar ações no PET-Saúde. Uma das barreiras encontradas foi o espaço físico, pois a maioria dos centros de saúde tinha espaço reduzido e com escassez de salas disponíveis e computadores. Os alunos também informaram que a carga horária semanal dedicada às ações na UBS (8 horas) era baixa e que a inserção de apenas um aluno por UBS dificultava a realização das atividades. Na visão deles, o aumento na quantidade de alunos para dois por UBS e a ampliação da carga horária semanal proporcionariam um nível mais elevado de autoconfiança na realização das ações, além de aumentar a quantidade e o escopo delas.

\section{Categoria: Habilidade de realizar ações de educação em saúde}

Dentre as atividades de integração ensino-serviço-comunidade realizadas pelos estudantes de Farmácia do PETSaúde/GraduaSUS, destacaram-se aquelas educativas. Os alunos se envolveram em ações de educação em saúde verbal e escrita.

Como os estudantes de Farmácia atuaram em várias UBS no PET-Saúde/GraduaSUS, eles sugeriram e criaram diversos materiais didáticos impressos que poderiam ser utilizados em todas as UBS do SUS em Belo Horizonte. Por exemplo, foram desenvolvidos um guia destinado a divulgar os serviços ofertados na UBS e um panfleto sobre funcionamento das farmácias e dispensação de medicamentos na APS em Belo Horizonte. As atividades de educação em saúde verbal foram realizadas em parceria com a equipe interprofissional das UBS e demais acadêmicos de diferentes cursos da área da saúde, com o emprego de metodologias ativas de aprendizagem, como dinâmicas de grupo, jogos educativos, dentre outras.

Foram desenvolvidas pelos alunos do PET-Saúde/GraduaSUS habilidades de busca de informações na literatura técnico-científica para conferir embasamento para o planejamento das atividades educativas. Os alunos compreenderam que na realização das ações de educação em saúde verbal são necessárias a priorização daquelas informações que são essenciais e o estabelecimento de uma forma de comunicação com o usuário que aumente a sua motivação, como ilustrado no relato do aluno abaixo. 
[...] Na tenda [tipo de atividade educativa] você vai tentar captar as pessoas e falar algumas informações que interessam, que são mais importantes, informações que interessam mais para aquela pessoa (Aluno 8 em trecho de entrevista)

Em um modelo para o estabelecimento de educação verbal em saúde efetiva, Marcus (2014) propõe o fornecimento de atividades educativas centradas no paciente, considerando as visões e preferências deste, enfatizando de uma a três ideiaschave e empregando uma linguagem de fácil entendimento. Os graduandos do PET-Saúde/GraduaSUS consideraram estes aspectos no planejamento e realização das atividades educativas.

\section{Limitações}

Como toda pesquisa científica, o presente estudo apresenta limitações. A coordenadora do grupo tutorial de Farmácia do PET-Saúde/GraduaSUS realizou as entrevistas, o que pode ter inibido os estudantes de realizar comentários sobre a coordenação do grupo. A coleta de dados ocorreu ao término do projeto, sendo que para próximos estudos seria interessante analisar as percepções dos graduandos antes e após a aprendizagem experiencial.

\section{Conclusão}

Os relatos dos estudantes do curso de Farmácia sugerem que os objetivos de aprendizagem propostos para a participação no PET-Saúde/GraduaSUS foram alcançados. O Projeto PET-Saúde possibilitou a oportunidade de formação acadêmico-profissional no âmbito interprofissional e na atenção e cuidado à saúde. Esta experiência possibilitou aplicar os conhecimentos adquiridos em sala de aula e na comunidade, enriquecendo a formação acadêmica e contribuindo para a humanização da profissão, mudando a forma como o profissional farmacêutico é visto, permitindo um olhar mais amplo sobre as potencialidades de sua atuação, transferindo o foco central de profissional do medicamento para um profissional dedicado também à atenção ao paciente, consolidando-se como uma forma de atuar na vigilância e na promoção e prevenção da saúde.

O projeto permitiu uma imersão na realidade do SUS, durante a graduação, por meio da convivência com diversos setores e serviços da área da saúde, profissionais e usuários, levando a uma troca de experiências e mútua aprendizagem, contribuindo para futura atuação profissional comprometida e de acordo com os princípios e diretrizes do SUS. O PET- Saúde é um instrumento de indução de mudanças nas concepções dos profissionais de saúde, egressos e acadêmicos, possuindo grande potencial para apoiar na formação sólida, generalista, reflexiva e humanista preconizada para os cursos de saúde. Recomenda-se o fomento e o aperfeiçoamento deste programa, sobretudo, a expansão do mesmo para um número maior de estudantes, para garantir a efetividade das ações propostas. Para próximos estudos, recomenda-se uma análise das percepções dos graduandos antes e após a aprendizagem experiencial, empregando-se uma combinação de métodos quantitativos e qualitativos.

\section{Agradecimentos}

Agradecemos ao Ministério da Saúde pelo financiamento das bolsas do Programa PET-Saúde/GraduaSUS e ao Programa de Iniciação Científica Voluntária (ICV) da Universidade Federal de Minas Gerais, do qual a autora Thaissa Rocha Dubón fez parte.

\section{Referências}

Agreli, H. F.; Peduzzi, M.; Silva, M. C.; Mascarelle, R. C. V \& Espinoza, P. (2019). Efeito da educação interprofissional no trabalho em equipe e no conhecimento do manejo de condições crônicas. Revista Latino-Americana de Enfermagem, 27, e3203. 
Bardin, L. (2002). Análise de conteúdo. Lisboa: Edições 70.

Barreto, I. C. H. C.; Loiola, F. A.; Andrade, L. O. M.; Moreira, A. E. M. M.; Cavalcanti, C. G. C. S.; Arruda, C. A. M. \& Silva, A. L. F. (2011). Development of interprofessional collaborative practices within undergraduate programs on healthcare: case study on the Family Health Alliance in Fortaleza (Ceará, Brazil). Interface-Comunicação, Saúde, Educação, 15 (36):199-212.

Brasil. Ministério da Saúde. (2015). Edital n 13 de 28 de setembro de 2015. Seleção para o Programa de Educação pelo Trabalho para a Saúde PETSaúde/GraduaSUS-2016/2017. Diário Oficial da União. Brasília, 29 set. 2015; Seção 3, p.126.

Brasil. Ministério da Saúde. (2017). Portaria nº 2.436, de 21 de setembro de 2017. Aprova a Política Nacional de Atenção Básica, estabelecendo a revisão de diretrizes para a organização da Atenção Básica, no âmbito do Sistema Único de Saúde (SUS). https://bvsms.saude.gov.br/bvs/saudelegis/gm/2017/prt2436_22_09_2017.html

Destro, D. R.; Martins, B. B. R.; Brito, M. J. M. \& Chemello, C. (2021). Perspectivas de pacientes sobre o acompanhamento farmacoterapêutico na Atenção Primária à Saúde em uma capital brasileira. Research, Society and Development, 10(9): e37510918125.

Filho, J. R. F.; Silva, C. B. G.; Costa, M. V. \& Forster, A. C. (2019). Educação Interprofissional nas políticas de reorientação da formação profissional em saúde no Brasil. Saúde em Debate, 43(spe1): 86-96.

FIP. International Pharmaceutical Federation. (2012). FIP Education Initiatives: Pharmacy Education Taskforce. A Global Competency Framework. The Hague, Netherlands: International Pharmaceutical Federation. https://www.fip.org/file/1412

Fonseca, G. S.; Junqueira, S. R.; Zilbovicius, C. \& Araujo M. E. (2014). Educação pelo trabalho: reorientando a formação de profissionais de saúde. InterfaceComunicação, Saúde, Educação, 18(50):571-583.

Frank, J. R.; Snell, L. S.; Cate, O. T.; Holmboe, E. S.; Carraccio, C.; Swing, S. R.; Harris, P.; Glasgow, N. J.; Campbell, C.; Dath, D.; Harden, R. M.; Lobst, W.; Long, D. M.; Mungroo, R.; Richardson, D. L.; Sherbino, J.; Silver, I.; Taber, S.; Talbot, M. \& Harris, K. A. (2010). Competency-based medical education: theory to practice. Medical Teacher, 32(8):638-645.

Kairuz, T.; Noble, C. \& Shaw, J. (2010). Preceptors, interns, and newly registered pharmacists' perceptions of New Zealand pharmacy graduates' preparedness to practice. American Journal of Pharmaceutical Education, 74(6):108.

Kolb, D. A. (2015). Experiential learning: Experience as the Source of Learning and Development. Upper Saddle River, NJ: Pearson Education.

Kwon, J.; Kim, J. J.; Koval, P. G. \& Hammer, S. K. (2021). Intervention documentation of second- to fourth-year pharmacy students during clinical experiential rotations. Currents in Pharmacy Teaching and Learning, 13(3):266-272.

Leite, M. T. S.; Rodrigues, C. A. Q.; Mendes, D. C.; Veloso, N. S.; Andrade, J. M. O. \& Rios, L. R. (2012). O Programa de Educação pelo Trabalho para a Saúde na Formação Profissional. Revista Brasileira de Educação Médica, 36 (1suppl1): 111-118.

Lima, M. G.; Ceccato, M. G. B.; Braga, D. S.; Silva, F. M. B.; Gonçalves, M. A.; Gajo, M. M.; Rebuitti, R. B.; Silveira, M. R.; Dias, A. A. M.; Casula, D. A.; Nascimento, I. R. \& Mendes, D. F. (2014). Grupos operativos de hipertensos e diabéticos no pet-saúde. Revista Brasileira de Pesquisa em Saúde, 16(1):133-8.

Mangueira, S. O.; Macedo, E. M. C.; Albuquerque, V. L. R.; Pereira, M. C.; Nascimento, E. C. L.; Freitas, M. A. A.; Souza, G. W.; Pinheiro, A. L. L. S.; Tardieux, F. M.; Silva, J. B. O.; Júnior, L. S. M.; Barbosa, D. A. M. \& Santos, C. S. (2021). A prática colaborativa na formação em saúde: Relato de experiência do PET saúde Interprofissionalidade. Research, Society and Development, 10(5): e9110514565.

Marcus, C. (2014). Strategies for improving the quality of verbal patient and family education: a review of the literature and creation of the EDUCATE model. Health Psychology \& Behavioural Medicine, 2(1): 482-495.

Marriott, J. L., Nation, R. L., Roller, L., Costelloe, M., Galbraith, K., Stewart, P \& Charman, W. N. (2008). Pharmacy education in the context of Australian practice. American Journal of Pharmaceutial Education, 72(6), 131.

Mendes, S. S., Cardoso, J. S., Groia, R. C. S., Braga, D. S., Sorrentino, F., Silva, F. M. B., Gajo, M. M., Gonçalves, M. A., Camargos, R. C., Rebuitti, R. B., Garcia, G. A., Casula, D. A., Corradi, C. O., Ceccato, M. G. B., Silveira, M. R. \& Lima, M. G. (2014). Contributions to the pharmaceutical assistance: report of an experience in the PET-Health Program. Revista Médica de Minas Gerais, 24 (Supl.1), S19-S24.

Mendonça, S. A. M.; Freitas, E. L. \& Oliveira, D. R. (2017). Competencies for the provision of comprehensive medication management services in an experiential learning project. PLoS ONE, 12(9):e0185415.

Nemire, R. E. \& Meyer, S. M. (2006). Educating student for practice: educational outcomes and community experience. American Journal of Pharmaceutial Education, 2006;70(1):20.

Peduzzi, M.; Norman, I. J.; Germani, A. C. C. G.; Silva, J. A. M. \& Souza, G. C. (2013). Educação interprofissional: formação de profissionais de saúde para o trabalho em equipe com foco nos usuários. Revista da Escola de Enfermagem da USP, 47(4): 977-83.

Pimentel, A. (2007). A teoria da aprendizagem experiencial como alicerce de estudos sobre o desenvolvimento professional. Estudos de Psicologia, 12(2): $159-168$.

Reeves, S. (2016). Porque precisamos da educação interprofissional para um cuidado efetivo e seguro. Interface-Comunicação, Saúde, Educação, 20(56): 185196.

Rosa, R. P. F.; Andrade, A. L. F.; Oliveira, S. P.; Silva, A. G. L.; Ferreira, A. M.; Inácio, J. S. \& Araújo, S. M. S. S. (2015). Construindo saberes no trabalho em saúde mental: experiências de formação em saúde. Interface-Comunicação, Saúde, Educação, 19(suppl1): 931-940. 
Research, Society and Development, v. 10, n. 17, e46101724277, 2021

(CC BY 4.0) | ISSN 2525-3409 | DOI: http://dx.doi.org/10.33448/rsd-v10i17.24277

Silva, J. A. M.; Peduzzi, M.; Orchard, C. \& Leonello, V. M. (2015). Educação interprofissional e prática colaborativa na Atenção Primária à Saúde. Revista da Escola de Enfermagem da USP, 49(spe2):16-24.

Turhan, N. S. (2019). Qualitative research designs: which one is the best for your research? European Journal of Special Education Research, 4(2): 124-136. 